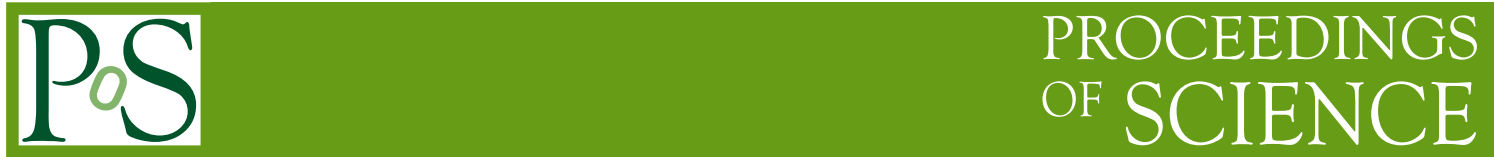

\title{
In Search of Exomoons
}

\section{David M. Kipping ${ }^{* \dagger}$}

Harvard-Smithsonian Center for Astrophysics, Cambridge, MA, USA

E-mail: dkipping@cfa.harvard.edu

Two decades ago, astronomers began detecting planets orbiting stars other than our Sun, so-called exoplanets. Since that time, the rate of detections and the sensitivity to ever-smaller planets has improved dramatically with several Earth-sized planets now known. As our sensitivity dives into the terrestrial regime, increasingly the community has wondered if the moons of exoplanets may also be detectable, so-called "exomoons". Their detection represents an outstanding challenge in modern astronomy and would provide deep insights into the uniqueness of our Solar System and perhaps even expand the definition of habitability. Here, I will briefly review theoretical studies exploring the formation and evolution of exomoons, which serve to guide observational searches and provide testable hypotheses. Next, I will outline the different methods which have been proposed to accomplish this challenging feat and their respective merits. Finally, initial results from observational efforts will be summarized with a view to future prospects as well.

Frank N. Bash Symposium 2013: New Horizons in Astronomy (BASH 2013)

October 6-8, 2013

Austin, Texas

\footnotetext{
* Speaker.

${ }^{\dagger}$ NASA Carl Sagan Fellow.
} 


\section{INTRODUCTION}

Within the field of astronomy, there are few areas of research which have enjoyed as much public enthusiasm and rapid rates of discovery than that of exoplanetary science - a research area which was not even established until two decades ago. Astronomers first waded out around the shores of this novel discipline around 1990, with compelling evidence for exoplanets found by [1], [2] and [3]. However, it was not until the mid-nineties, after the discovery of 51 Pegasi b by [4], that astronomers unfurled their sails and began regularly ensnaring impressive hauls of exoplanets.

Since then, more than one thousand confirmed exoplanets and five thousand candidates have been detected. Furthermore, observational sensitivity has dramatically improved from being limited to hot-Jupiters back in the mid-nineties [5] to now being able to detect Earth-sized habitablezone worlds [6]. This progress stems from a wide range of improvements in the instrumentation, telescope time, use of dedicated missions, man-power, observing strategy, modeling and of course research funding, which enables all of the above [7].

This journey of scientific discovery is surely not complete though, and many mysteries and outstanding challenges remain. For example, we now know that our solar system and our planet represent just a drop in the cosmic ocean, and the surprising diversity of planetary architectures and compositions indicates that we lack the deep understanding of how planets form and evolve [8]. Similarly, whilst empirical estimates of the occurence rate of habitable-zone rocky planets have been made $[9,10]$, the detection of bio-signatures or detailed atmospheric characterization of such worlds is still some way off [11].

With our sensivity to exoplanets reaching the level of Earth and even sub-Earth sized worlds [6], one outstanding question which may be within reach is the detection of extrasolar moons; so-called "exomoons". Their detection would likely usher in an entire new sub-field of scientific endeavor, much like the wind change instigated by the discovery of 51-Pegasi-b. Measuring the physical and orbital characteristics of ensemble populations of exomoons would likely yield powerful constraints on the formation and evolution mechanisms guiding alien worlds [12]. Such bodies may themselves be habitable $[13,14,15,16]$ or affect the habitability of the planets which they orbit [17]. A confirmed exomoon detection would therefore represent a major scientific breakthrough, yet this lofty goal remains a daunting and outstanding challenge to modern astronomy.

In this work, I will briefly discuss several plausible formation mechanisms for exomoons in $\S 2$, with a view as to how these ideas may impact observational strategies. In $\S 3$, I will review the proposed detection techniques and sensitivities, with focus on the transit-based methods. In $\S 5$, empirical results to date will be summarized and placed into context. Finally, in $\S 6$, we discuss future prospects for exomoon detection with current and planned exoplanet missions. 


\section{FORMATION \& EVOLUTION}

\subsection{Context}

We begin by briefly discussing several possible formation and subsequent evolution mechanisms for exomoons. Since we currently know of no confirmed exomoons, this topic is strongly influenced by inspection of the moons within our solar system, so-called "endomoons". Given the enormous diversity of exoplanetary architectures discovered to date, which rarely resemble the Solar System, it is important to not put too much faith in such theories. Nevertheless, they provide a useful starting point and at least offer hypotheses which can be subsequently tested with observations. I therefore endeavor to provide only a brief introduction to give a flavor of how theory could guide observing strategy.

In our solar system, we observe two broad classes of satellites, although there exists no widely accepted definition as to what divides these two classes. In this work, I define regular satellites to have formed in-situ from the circumplanetary disk and these tend to display nearly-coplanar and nearly-circular orbits. In contrast, irregular satellites are those which formed via some other mechanism, such as an impact or a capture, and these tend to exhibit highly inclined (including retrograde motion) and highly eccentric orbital paths. Regular satellites also tend to have more compact orbits than the irregular population. Naturally, these two populations have distinct histories and thus I will split the discussion of formation and evolution accordingly.

\subsection{Formation of Regular Satellites}

Although considerable diversity exists within the literature for proposed formation mechanisms of the regular satellites, the fact that such satellites have compact and prograde orbits has led to the general consensus that they formed from a circumplanetary disk [18]. In general, formation models focus on the Jovian (belonging to Jupiter) and Kronian (belonging to Saturn) satellites, since these two planets host the largest number of regular satellites and have enjoyed a long history of detailed observations. In this framework, the primary challenge is to understand the differences between the number of major moons.

One of the leading formation models comes from a series of papers developed by [19, 20, 21] and is known as the actively supplied gaseous accretion disk model. In this model, dust grains within a circumplanetary disk stick and grow to form satellitesimals, which then migrate via type I migration and are disposed within $\sim 10^{5}$ yrs [22]. Continuous mass-infall form the protoplanetary disk maintains a peak circumplanetary disk density of $\sim 100 \mathrm{~g} \mathrm{~cm}^{-2}$, allowing new satellitesimals to continuously grow. Once the planet has opened up a gap in the protoplanetary disk, the active supply halts and the circumplanetary disk rapidly diffuses in $\sim 10^{3} \mathrm{yrs}$, thus "freezing" the remaining satellites in place. The final total satellite mass is therefore given by a balance between type I migration disposal and the repeated satellitesimal accretion rate, which [19] argue to be universally of order $\left(\sum M_{S}\right) / M_{P} \sim 10^{-4}$. In this model, the Jovian/Kronian differences are proposed to be due to presence of an inner cavity within the circum-Jovian disk, which Saturn was unable to form due its distinct mass and semi-major axis [23].

An alternative model comes from [18, 24] and [25]: the solids enhanced minimum mass model. Here, the planet hosts a two-component disk comprised of a dense inner sub-disk surrounded by a lower density outer disk. This results in a much longer satellite migration timescale 
than the associated formation timescale. The model qualitatively describes the expected mass ratios, but does not provide definitive predictions- unlike the actively supplied disk accretion model.

\subsection{Formation of Irregular Satellites}

In this Review, I consider irregular satellites to be those which initially formed not as a satellite to the planet in question, but somehow ended up so via a capture or impact event. Before this event, the satellite may have been a planet/dwarf planet itself, a Trojan, a satellite of another planet or a binary pair. For moons to form via a capture or impact, one clearly requires strong dynamical mixing leading to close encounters. The ultimate outcome of an encounter will chiefly depend upon how close these encounters are (the impact parameter) and the relative masses and velocities of the bodies involved. In this sense, one can consider impacts to be a special case of a capture, where the impact parameter of the event is less than one planetary radius.

Giant planets must frequently migrate given the observed population of hot Jupiters, which certainly could not have formed in-situ [26]. During this inward migration, the giant planet may encounter terrestrial planets and in such encounters a capture could transpire. In all capture scenarios, one requires the relative velocities of the two bodies to be below that of the planet's escape velocity, if the putative satellite is to be captured. This generally requires a deceleration of the putative satellite, for which several mechanisms have been proposed.

In the case of the Moon, our satellite's composition, rotation and orientation support the socalled "giant impact hypothesis" between the primordial Earth and a Mars-sized body some $4.5 \mathrm{Gyr}$ ago [27]. Whilst this hypothesis explains much of the available evidence, it is unclear how frequently such events may occur or how special the Moon may be. Indeed, this question strongly motivates seeking observational evidence for exomoons.

A mechanism for producing irregular moons, requiring a less finely tuned impact parameter, comes from the "binary-exchange mechanism" [28]. Here, a binary of similar masses encounters a giant planet, causing tidal disruption of the binary. If the binary has the correct sense of revolution, then the member closest to the giant planet can be strongly decelerated by virtue of the binary's interior motion. This body is then captured to an initially highly inclined and/or eccentric orbit, whilst the other member of the binary is ejected. This hypothesis has gained favor for the origin of Triton and [29] have suggested that for exomoons, mass ratios up to 10:1 may result.

Once a moon has been initially captured, the dynamically hot orbit is rapidly circularized via tides. [30] estimate this process occurs on a timescale of $10^{3}$ yrs leading to $25-60 \%$ of satellites surviving into circularized, stable orbits. Irregular satellites therefore offer an apparently plausible mechanism for the origin of large moons, such as the Moon and Triton.

\subsection{Likely Formation of Detectable Exomoons}

Current theories of satellite formation therefore indicate that the highest satellite-to-planet mass ratio moons are more feasibly formed via a capture/impact event, rather than precipitating out of the circumplanetary disk. This point is crucial since current observational searches for exomoons are only sensitive to high mass ratios exomoons of order of $1 \%$ to $10 \%$, as will be discussed later. As seen in the next subsection, an irregular moon can in fact have a longer survival time in the subsequent orbital evolution too. On this basis, current models imply that any exomoons large enough to be detected in the near future are more likely to be irregular moons. 
A large mass-ratio exomoon captured around an exoplanet is presumably a relatively rare event. The Moon-Earth system presents the only known example of a $\mathscr{O}\left[10^{-2}\right]$ mass-ratio in the Solar System (ignoring the dwarf planets) leading to the conclusion that the conditions under which the Moon formed were certainly not typical for all endoplanets. Determining the occurence rate of such large mass ratio exomoons will of course be an important measurement from observational searches to constrain current theories. Producing these events requires either a very low impact parameter or a low velocity encounter between two bodies [29], for which either scenario seems to be the tail-end of the probability distribution of possible outcomes. With these arguments in mind, it would seem that likely that in the apparently rare instances where a moon has a $\mathscr{O}\left[10^{-2}\right]$ mass-ratio to the host planet, it is likely the only significant exomoon in orbit of said planet. For reasons discussed in the last subsection, I here posit that any exomoon detected in the near future will not co-exist with any other major satellites. Accordingly, moon-moon interactions will not significantly feature in the subsequent orbital evolution.

\subsection{Long-Term Evolution of Exomoons}

For an irregular satellite, once the initial rapid tidal circularization has completed in just a few thousands years, the subsequent orbital evolution of the moon is dominated by tidal interactions with the host planet and gravitational interactions with other satellites in the system. For reasons discussed in the last subsection, I here consider that any detectable exomoon in the near-future will not be competing with any other major satellites and thus moon-moon interactions will not significantly feature in the subsequent orbital evolution.

The only mechanism which therefore contributes significantly to the orbital evolution of our exomoon is tidal dissipation. Consider a planet-moon pair with a near-circular orbit and rotation periods shorter than the revolution period. Here, the tidal evolution occurs in three stages. Firstly, the torque applied by the planet's gravity on bulges induced on the moon leads to tidal friction and a gradual decrease in the rotation rate of the satellite, ultimately leading to tidal locking of the satellite. By conservation of angular momentum, the orbit of the satellite is slightly raised by this process. Next, the same tidal torque slows down the rotation of the planet over a longer timescale and again lifts the orbit of the satellite. Finally, the planet's rotation rate becomes synchronized with the revolution rate so both bodies are tidally locked into a spin-orbit resonance and after this point tidal evolution ceases. For the Earth-Moon system this is some $50 \mathrm{Gyr}$ away and would occur at a period of $47 \mathrm{~d}$. In practice, a slight drag force on the satellite would decay the orbit until the tidal evolution kicks in again, but now in reverse, causing inward migration, since now the position of the tidal bulge trails the planet-moon axis.

[31] modeled this process for a variety of planet-moon pairs and found that even for habitablezone planets, the Hill sphere (the volume of stable moon orbits) is large enough that losing a moon through tidal evolution takes many Gyr. [32] argue the maximum orbital radius for stable moon orbits is actually about twice as large for retrograde satellites, suggesting they should have much longer tidal lifetimes than their prograde counterparts. In conclusion, the process of tidal evolution for a planet-moon pair seems unlikely to be a significant bottleneck to a present day population of large exomoons. In contrast, the initial formation of such moons seems to require some fine tuning. Therefore, the very presence of a large exomoon would be interesting from a formation perspective while the present-day orbital parameters would be interesting from an evolution perspective. 


\subsection{Stripping a Planet of its Moons}

For a single star with just one circular orbit planet which harbors a single moon, orbital evolution should indeed proceed as described above. In reality, additional bodies and eccentricity can evolve the planet's orbit and lead to scattering events. If a planet with a moon migrates inwards through the protoplanetary disk in the first few million years after formation, the Hill sphere of the planet will shrink much more rapidly than the timescale for orbital evolution of the moons. This causes any moons to essentially find themselves suddenly outside the Hill sphere and thus ejected, as discussed by [33]. The author suggests giant planets which have migrated interior to $0.1 \mathrm{AU}$ will have lost most of their initial moons by this process. For this reason, observational searches should likely avoid hot-Jupiters systems.

[12] recently discuss how solar systems with just a single planetary member may imply a history of severe planet-planet scattering. Close encounters between planets is found to efficiently strip the planets of their moons, although in some cases exchanges of satellites may occur. For this reason, [12] recommend observational searches focus on multi-planet systems. In practice, it is practically impossible to determine that a star absolutely only hosts one planet, especially for transiting systems, and so this advice should be taken with some caution.

\section{DETECTION TECHNIQUES}

\subsection{Overview}

Numerous techniques for detecting exomoons have been proposed since their planetary counterparts began to be found. Here, I will focus exclusively on those detections methods which rely on the transit technique - that is, when an exoplanet periodically passes in front of the host star causing a repeated decrease in the apparent brightness. Transits only occur for planets which happen to have nearly edge-on orbits relative to our line of sight. For a circular orbit planet, the geometric probability is simply the radius of the star divided by the semi-major axis and is $\mathscr{O}\left[10^{-2}\right]$. Despite this drawback, the transit method has emerged has one of the dominant techniques for discovering exoplanets and arguably the dominant technique for exoplanet characterization.

The reason why the transit method has enjoyed so much focus, funding and interest from the community is perhaps down to the simplicity of the observation and the rich plethora of planet properties which can inferred. As an example, the shape of the transit light curve encodes the inclination of the orbit [34], which can be combined with a radial velocity (RV) signal to back out the planet's true mass (rather than just the minimum mass, which RVs usually provide). Characterizing the atmospheres of exoplanets has sprung forth an entire field of scientific activity, usually by seeking changes in the depths of transits in different wavelengths, betraying the presence of absorbing molecules in the atmospheres [35].

Eclipsing bodies have a long history of providing deep physical insights and sensitivies and the cost of fewer objects to study [36]. It is for similar reasons that the transit method provides viable methods for detecting exomoons. However, one should proceed under no illusion that this is by any means easy, even when leveraging the unique insights provides by transits.

Other methods for detecting exomoons include pulsar timing [37], which is limited to looking at a relatively unusual type of star. Whilst a direct image of an exomoon is some way off given 
their likely tiny size, it has been argued that evidence for circumplanetary disks has been captured in exoplanet direct imaging [38]. The microlensing technique is also capable of finding exomoons and has even reported a possible candidate recently, around a free-floating planet [39]. However in general, and certainly in the case of this candidate, it is not possible to uniquely determine the mass of the lens pair, meaning that a low-mass star with a planet is also a viable explanation to the observations [39]. Additionally, microlensing events are a-periodic and thus there is no prospect of future observations to confirm any claimed detections.

In conclusion, I would argue that the transit method is the favored technique for discovering exomoons, allowing one to i] see repeated events and make causal predictions regarding future observations (a fundamental pillar of the scientific method) ii] detect exomoons around any luminous star which has a transiting planet iii] characterize any discoveries with future observations to measure the atmosphere and orbit iv] determine all of the basic physical parameters of the moon from time series photometry alone. In the following subsections, I will therefore focus exclusively on the transit method and discuss how the claimed feats mentioned above are possible.

For transiting systems, there are two broad class of observable effects due to an exomoon: dynamical effects and eclipse effects. I will discuss each of these in the following subsections.

\subsection{Dynamical Effects}

\subsubsection{Transit Timing Variations (TTV)}

The first scientific paper to propose a method for detecting the moons of exoplanets suggested seeking variations in the times of the transit events [40]. Further, this paper appears to be the first usage of the term "transit timing variations" (TTV), which became lately more commonly associated with detecting planet-planet gravitational interactions [41, 42]. As shown in Figure 1, a planet with a moon will exhibit reflex motion in response to the companion's mass on top of the usual Keplerian orbit around the host star. This reflex motion causes changes in the position and velocity of the planet relative to a strict Keplerian, and these changes manifest as transit timing variations (TTV) and transit duration variations (TDV-V) respectively. It is worth noting that conceptually TTV is similar to the asterometric method of finding planets, whereas TDV-V is similar to that of the radial velocity method. Whilst [40] derived the expected TTV amplitude for circular orbits, the more general eccentric case was found by [43] to be given by:

$$
\delta_{\mathrm{TTV}}=\frac{a_{S} M_{S}}{a_{P} M_{P}} P_{P} \frac{\left(1-e_{S}^{2}\right) \sqrt{1-e_{P}^{2}}}{\left(1+e_{P} \sin \omega_{P}\right)} \sqrt{\frac{\Phi_{\mathrm{TTV}}}{2 \pi}},
$$

where $\delta_{\mathrm{TTV}}$ is the root-mean-square (RMS) TTV amplitude, $a_{S}$ and $a_{P}$ are the planet-satellite and planet-star semi-major axes, $M_{S}$ and $M_{P}$ are the satellite and planet masses, $e_{S}$ and $e_{P}$ are the orbital eccentricities of the satellite and the planet, $\omega_{P}$ is the argument of periastron of the planet and $\Phi_{\mathrm{TTV}}$ is absorbs many of the effects of orbital eccentricity and is defined in [43]. The effect is typically of order of seconds to minutes for terrestrial moons around gas giant planets, which is comparable to Kepler's timing precision.

From Equation 3.1 and ignoring eccentricity, one can see that the amplitude scales as $\sim M_{S} a_{S}$ i.e. $M_{S}$ and $a_{S}$ are fully degenerate. In principle, one could determine the period of the satellite, $P_{S}$, 


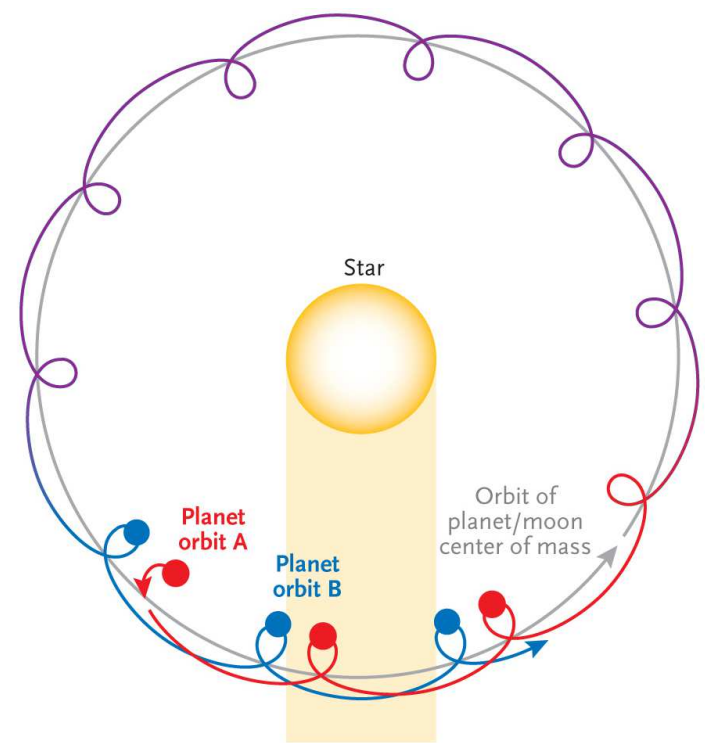

Figure 1: Schematic illustrating the (exaggerated) motion of a planet around its host star in response to a massive companion satellite (not shown). The planet's position and velocity deviate from that expected from a simple Keplerian (gray line), producing variations in the times and durations of the transits, respectively.

by the periodicity of the TTV signal, which could then be converted to $a_{S}$ via Kepler's Third Law. However in practice this is not possible, since the moon's period must always satisfy $P_{S} \leq P_{P} / \sqrt{3}$ [44] for dynamical stability, which is below the Nyquist frequency. Therefore, a unique solution for $P_{S}$, and thus $a_{S}$, is not possible. Crucially then, the TTV RMS amplitude, which is simply found by measuring the scatter in the data, is all we can infer and this displays a complete degeneracy between the moon's mass and orbital separation.

This problem with using TTVs to detect exomoons is compounded by the fact planet-planet interactions can also induce confounding TTV signatures [41, 42], which would be a source of false-positives. For these reasons, TTV is generally unable to uniquely detect an exomoon. This motivated the community to develop alternative or complementary techniques to solve these issues.

\subsubsection{Velocity induced Transit Duration Variations (TDV-V)}

As discussed earlier, the reflex motion of the planet, in response to the moon, causes changes in the velocity of the planet, as well as the position. Velocity changes lead to variations in the durations of the transits $[44,45]$, which can present a detectable signal; so-called velocity-induced transit duration variations (TDV-Vs). The RMS amplitude of the TDV signal is given by [43]:

$$
\delta_{\mathrm{TDV}-\mathrm{V}}=\tilde{T}_{B}\left(\frac{a_{S} M_{S} P_{P}}{a_{P} M_{P} P_{S}}\right)\left(\frac{\sqrt{1-e_{P}^{2}} \sqrt{\Phi_{\mathrm{TDV}-\mathrm{V}} / 2 \pi}}{\sqrt{1-e_{S}^{2}}\left(1+e_{P} \sin \omega_{P}\right)}\right),
$$

where $\tilde{T}_{B}$ is the mean transit duration and $\Phi_{\mathrm{TDV}-\mathrm{V}}$ absorbs several effects due to orbital eccentricity and is defined in [43]. The effect is typically of order of seconds to minutes for terrestrial moons around gas giant planets, an amplitude similar to that of TTVs. Inspection of Equation 3.2 
reveals that the TDV-V amplitude scales as $\sim M_{S} a_{S} P_{S}^{-1}$. Recall that the TTV amplitude scales as $\sim M_{S} a_{S}$ though, meaning that $P_{S}$ (and thus $a_{S}$ via Kepler's Third Law) can be determined simply by comparing the TDV-V RMS amplitude to the TTV RMS amplitude (for circular orbits). This solves the undersampling issue encountered using TTVs in isolation.

An additional benefit of TDV-Vs, is that the planet-planet interactions do not usually produce detectable TDVs, and when they do the signal is expected to be either perfectly in phase or in antiphase $(\Delta \phi=0$ or $\pi)$, as seen in [46]. In contrast, the reflex motion of the planet is essentially twodimensional simple harmonic motion and thus the position (TTVs) and velocity (TDV-Vs) exhibit a $\Delta \phi=\pi / 2$ phase shift [44]. This specific signature enables the unique identification of exomoons. Despite the signals being undersampled, this phase shift can be inferred by cross-correlating the normalized squared TTVs and TDV-Vs [47].

Despite the mathematical elegance of combining TTVs and TDV-Vs, one important limitation is that we require the detection of both effects, which is in practice very challenging. One can easily see this by virtue of the amplitude scalings. Specifically, TTVs scale as $\left(M_{S} a_{S}\right)$ where as TDV-Vs scale as $\left(M_{S} a_{S}^{-1 / 2}\right)$. Therefore, moons close to the planet will cause a large TDV but a much smaller TTV, and vice versa. This means that in the absence of infinite signal-to-noise, only intermediate separation moons can be detected. [48] explored this issue in detail simulating a variety of planetmoon configurations and concluded that around one sixth of Kepler's target stars would provide sufficient signal-to-noise to detect both effects for Earth-mass moons with a $25 \%$ recovery rate. This calculation assumes various priors on the orbital elements for exomoons though, which one generally does not know since no Earth-mass moons exist in the Solar System. However, one can take the properties of the known Solar System moons and simulate their detectability using Kepler. To do so, one must move the orbits of the outer planets inwards, since the Kepler baseline only covers 4.35 years. Opting for a planetary period of $100 \mathrm{~d}$ then and using the typical Kepler timing uncertainties reported in [49], one can see in Figure 2 that several endomoons would be detectable.

\subsubsection{Transit Impact Parameter induced Transit Duration Variations (TDV-TIP)}

I briefly discuss an additional second-order TDV effect known as transit impact parameter induced transit duration variations, TDV-TIP [45]. So far, the TTV and TDV-V effects have ignored motion occurring out-of-the-plane. In reality, orbits exist in three-dimensions and the same positional variations which gives rise to translational shifts (leading to TTVs) will also have some non-zero component in the orthogonal direction. Inclination in either (or both) the planet and the satellite relative to the line-of-sight lead to the reflex motion slightly adjusting the "height" of the transit chord, or more specifically the transit impact parameter. Since the transit impact parameter strongly affects the transit duration [34], then TDVs ensue.

TDV-TIP is typically an order of magnitude lower amplitude than TDV-V, for nearly coplanar orbits [45], at the level of sub-seconds to seconds. In extremely inclined orbits, the signal can become dominant though and should always be accounted for when modeling timing signals. As proved in [45], their detection would allow for the inference of the sense of orbital motion of the moon i.e. whether it is prograde or retrograde. This determination would have important ramifications for understanding the origin of said exomoon, since regular moons do not form in retrograde orbits. It becomes clear, then, that the transit light curve of a planet-moon system contains infor- 


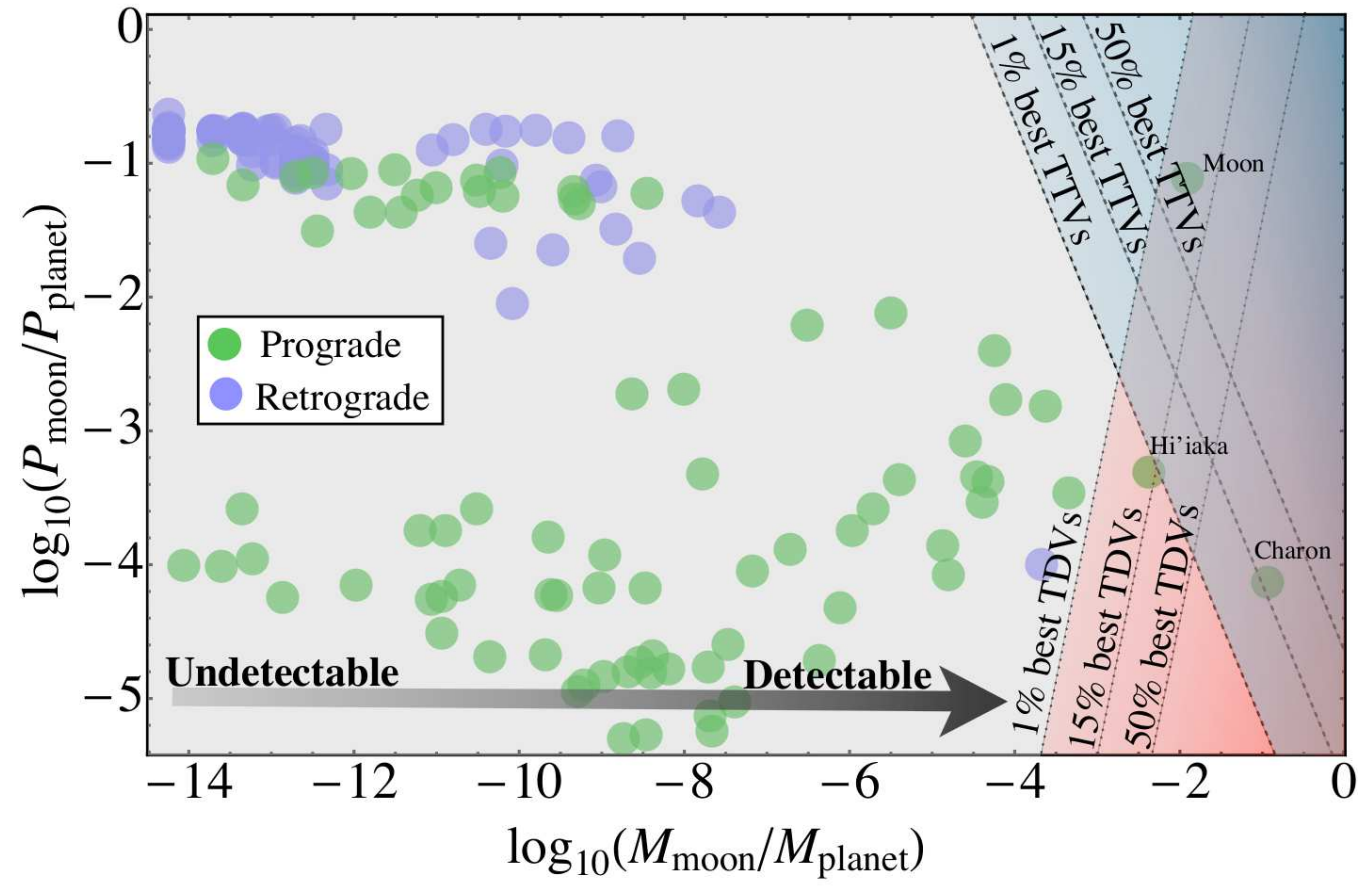

Figure 2: Period-ratio versus mass-ratio scatter plot of the Solar System moons. Transit timing and duration variations (TTV and TDV) exhibit complementary sensitivities with the period-ratio. Using the Kepler timing measurements from [49], one can see that the tip of the observed distribution is detectable. The above assumes a planetary period of $100 \mathrm{~d}$ and a baseline of 4.35 years of Kepler data.

mation on almost all of the orbital elements and thus once a system is identified, detailed follow-up could characterize these subtle effects.

\subsection{Eclipse Effects}

Although the direct transits of an exomoon may seem the most obvious way to detect such objects, efforts to model these events trailed behind the dynamical methods by a few years. Whereas the dynamical effects provide the mass of the satellite, eclipse effects would reveal the radius of the moon. Combining the two could then reveal the density of the satellite and thus inferences about the composition.

Eclipse effects come in two flavors; "auxiliary transits" and "mutual events". The former are simply those cases where the projected disk of the moon does not overlap with that of the planet but does overlap with that of the star. The latter is where all three disks overlap. These two effects have very different morphologies and modeling approaches.

\subsubsection{Auxiliary Transits}

In an auxiliary transit, the disk of the moon does not overlap with that of the star. This means one can simply compute the transit light curve profile caused by the planet and star separately add combine them together via: 


$$
I(t)=1-\left[\left(1-I_{P}(t)\right)+\left(1-I_{S}(t)\right)\right],
$$

where $I(t)$ is the overall normalized intensity of the star as a function of time, whereas $I_{P}(t)$ and $I_{S}(t)$ are the components due to the planet and satellite in isolation. These individual components may be simply computed using the standard [50], for example. [51] suggest that a planet with a very short-period could be folded to reveal excess scatter either side of the main transit event, due to auxiliary transits. Whilst simply seeking scatter would be a rapid way of detecting exomoons, it does not address the uniqueness of such an interpretation nor is it likely that short-period planets would exomoons anyway due to their much smaller Hill sphere.

\subsubsection{Mutual Events}

A mutual event is where all three disks overlap, meaning that one must compute the area of common overlap between the disks. This may be achieved using the analytic solutions from [52]. Typically, these events would occur by the planet and moon entering transit together with a small projected separation. The moon then skips across the face of the planet whilst both of the disks still overlap the star. During this time, the apparent flux of the star seems to increase, since one goes from having the planet and moon blocking the star to just the planet (since now the moon is hidden in front of or behind the planet). The moon then appears on the opposite side of the planet with a small separation and the two disks exit transit.

Examples of mutual events are shown in Figure 3. These events can look very similar to that of a planet passing over a dark starspot, leading to a potential source of false positive (e.g. see [53]). Mutual events can also, in some very rare instances, occur between two planets orbiting the same star [54]. Finally, it is worth noting that mutual events require a closely-aligned system for such events to be possible and a short-period/close-separation for such events to be frequent enough to expect to observe.

\subsection{Photodynamical Algorithms}

A photodynamical algorithm aims to model both the eclipse effects ("photo") and the dynamical effects simultaneously. Such codes have been leveraged in studying planet-planet interactions [55] and circumplanetary planets [56]. For exomoons, photodynamical algorithms don't merely serve to provide a better estimate of the system parameters but also fundamentally push one's sensitivity to small exomoons to the limit. This is because, in principle, a photodynamical algorithm models all of the observational imprints of an exomoon. Consequently, even if the significance of the TTVs, TDV-Vs, TDV-TIPs, auxiliary transits and mutual events are independently quite low, the combination of all these can push the significance above the discovery threshold. Since the field current lacks a confirmed exomoon detection, this point in fact represents the primary goal of the current generation of photodynamical algorithms - to enhance the act of detection.

With this goal in mind, practical considerations will also feature in the design of any such algorithm. For example, search techniques typically attempt billions of different possible starplanet-moon configurations and compare them to the data, in order to identify if the observations are well explained by including a moon or not. This is generally necessary due to multimodal 


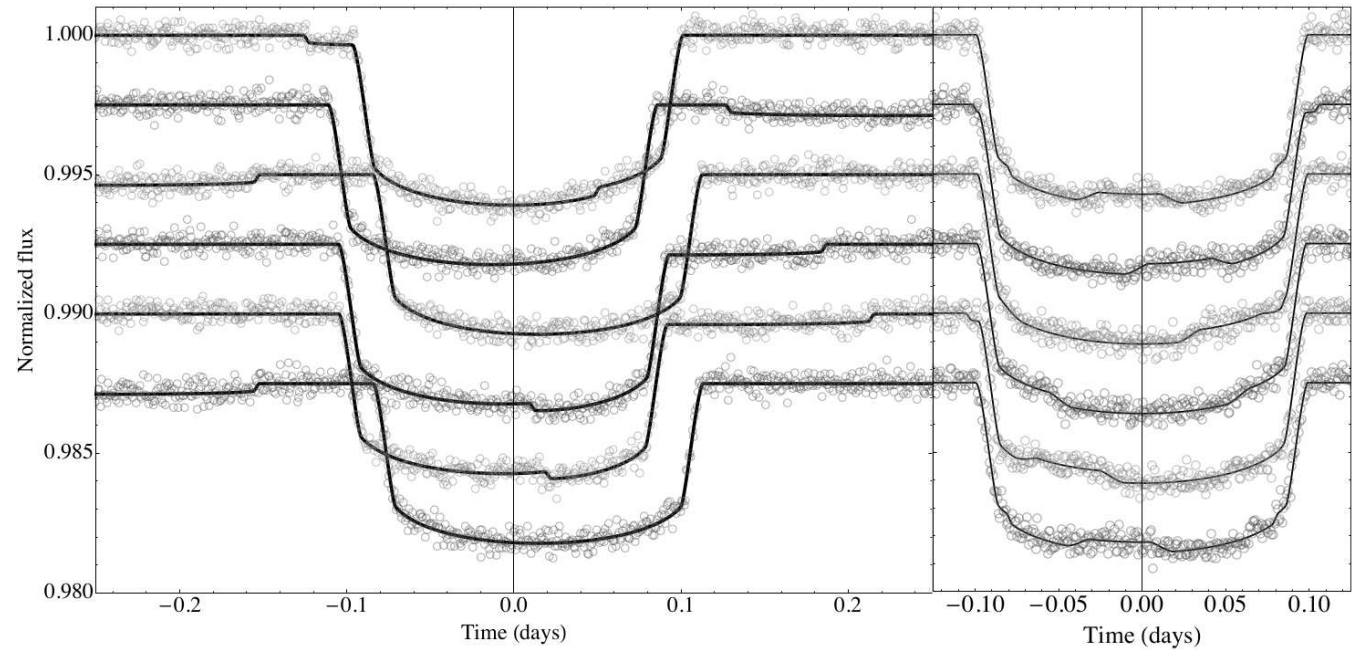

Figure 3: (Left) Six simulated transits using LUNA [62] of a HZ Neptune around an M2 star with an Earthlike moon on a wide orbit ( $90 \%$ of the Hill radius). The moon can be seen to exhibit auxiliary transits and induce TTVs. (Right) Same as left, except the moon is now on a close-in orbit (5\% of the Hill radius), causing mutual events. Both plots show typical Kepler noise properties for a $12^{\text {th }}$-magnitude star observed in short-cadence.

parameter space featuring complex and curved degeneracies [57], i.e. simple downhill algorithms are nearly useless for seeking evidence of exomoons. This requirement shapes the design of any algorithm since the model needs to be extremely fast to execute in order to make it feasible to scan multiple data sets for exomoons. Consequently, analytic photodynamical algorithms are the only practical solution.

Several approaches to producing a photodyanmical algorithm, to various degrees of completion, are available in the literature. [58] suggested re-defining the classic definition of the transit mid-time to a flux weighted "photo-center", which would incorporate some of the effects of both eclipses and dynamics. However, this approach does not account for orbital eccentricity or any other dynamical effects except TTV. [59,60] and [61] provide eclipse models which ignore the dynamical effects but reproduce the "photo" effects. Naturally, without a mass estimate of the moon it is unclear how reasonably one could uniquely identify signals as being due to an exomoon.

The only published full analytic photodynamical algorithm for exomoons, at the time of writing, is that of LUNA [62]. This code dynamically progress the position of the planet and moon at every time stamp, using a restricted three-body solution, and reproduces limb darkened light curves simulating both auxiliary and mutual transits. Simulations from LUNA are shown in Figure 3, illustrating the typical light curve morphologies of both auxiliary and mutual transits.

\section{RESULTS TO DATE}

In this section, I will focus on results published by the "Hunt for Exomoons with Kepler" (HEK) project [57]. To date, this is the only search project in the literature. HEK seeks to determine the occurrence rate of large moons around viable planet hosts, $\eta_{\mathbb{S}}$. 
The search is conducted using the photodynamical algorithm, LUNA, coupled with a multimodal nested algorithm called MultiNest [63, 64]. MultiNest is not only exhaustive, identifying all of the modes and complex curved degeneracies, but also provides the Bayesian evidence, $\mathscr{Z}$, of any model attempted. When one takes the ratio of $\mathscr{Z}$ between two models, say a planet-only versus a planet-with-moon model, the result is equal to the odds ratio between the models. In other words, we can determine which model is the most likely one. The Bayesian evidence penalizes models for using more parameters and thus includes a built-in Occam's razor. This is crucial when seeking exomoons, since including what is essentially a bunch of perturbation terms will always yield a better likelihood due to the higher number of degrees of freedom.

These Bayesian fits and parameters searches are computationally expensive though, taking up to 50 years of CPU time for a single system [65]. For this reason, HEK has been limited in the number of targets which can be investigated, focussing efforts on those planets which are thought to be capable of both maintaing moons via the tidal theory of [31] and presenting a detectable signal via the expected timing effects $[44,45]$.

\subsection{Constraints for 17 Exoplanets}

Spanning the papers $[66,67,65,68]$, a total of 17 Kepler planetary candidates have been surveyed by the HEK project, to date. There are no confirmed exomoons discoveries but upper limits on the mass ratio between a putative satellite and the host planet, $\left(M_{S} / M_{P}\right)$, in all cases, which are shown in Figure 4.
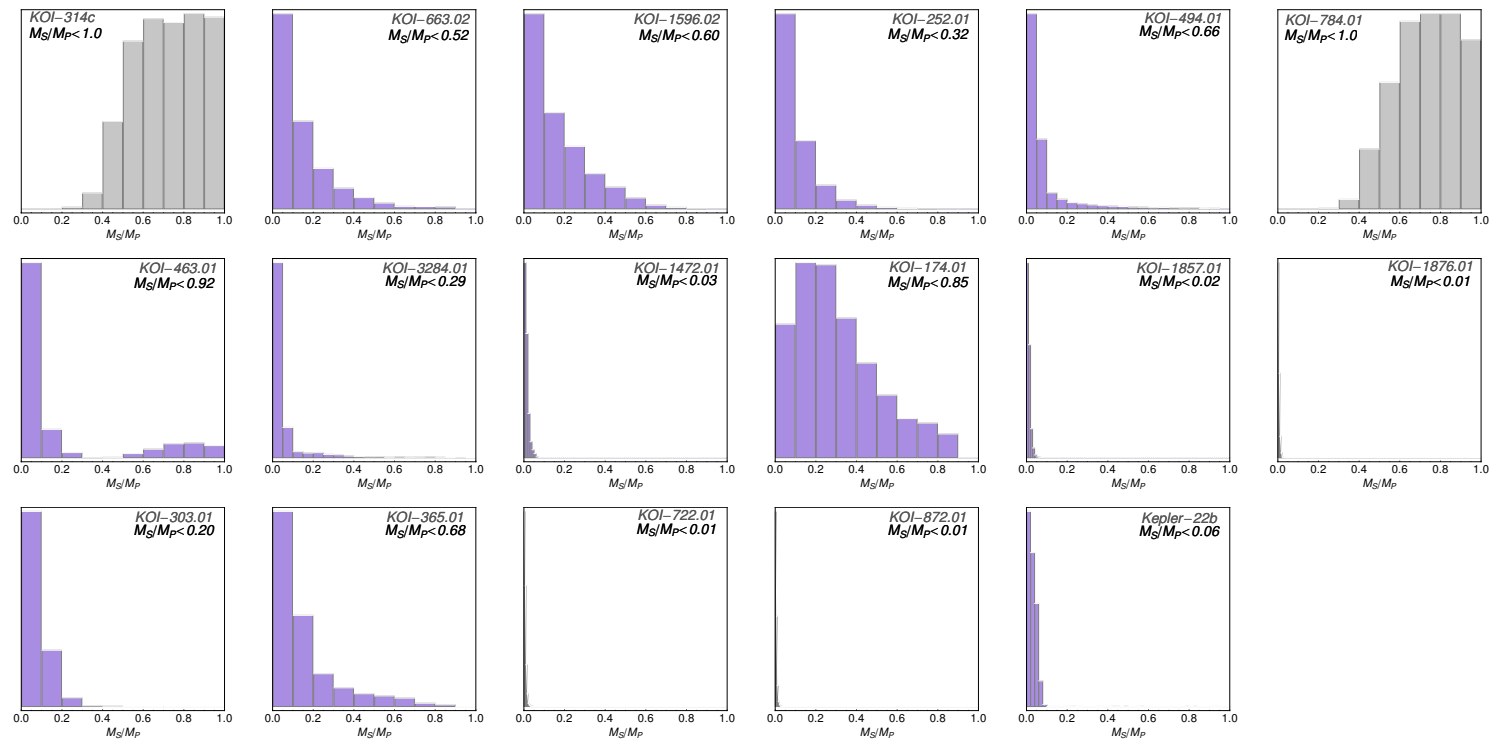

Figure 4: Empirical posterior distributions for the mass ratio between a putative satellite and 17 Kepler planetary candidates surveyed by the HEK project. 95\% confidence upper limits provided in the top-right. KOI-314c and KOI-784.01 are grayed out as planet-planet interactions lead to spurious detections.

Although the light curve fits only reveal $\left(M_{S} / M_{P}\right)$ directly, one can translate these values into physical masses by invoking a mass-radius relation for the planet. Using the [69] relation, six of the constraints correspond to sub-Earth mass sensitivity. [65] went further in the case of Kepler-22b by 
injecting a fake Earth-like moon into the observational data and retrieving it to $>8 \sigma$ confidence, demonstrating that HEK is certainly able to detect Earth-like moons should they exist. The absence of confirmed discoveries is therefore speaking to the underlying occurrence rate. At this point, the sample is clearly too small to derive a robust $\eta_{\overparen{S}}$ estimate, but this remains the ultimate goal of the HEK project.

\subsection{Ancillary Science}

Whilst the HEK project has probed down to sub-Earth masses and Earth-Moon mass ratios in its quest for exomoons, several interesting ancillary science discoveries have emerged from the project too. For example, [66] reported the first non-transiting planet discovered (with a unique orbit) via the TTV method in the KOI-872 system. This opens the door to not only discovering a new subset of planets hidden in the Kepler data, but also measuring precise masses and orbital elements in these dynamical systems. In this case, the TTVs were initially considered a plausible moon candidate but dynamical modeling revealed planet-planet interactions as the only viable model. [46] recently repeated the feat for another system, KOI-142, where the TTV amplitude was equal to nearly $10 \%$ of the orbital period, a situation the authors dubbed as "The King of TTVs".

[68] recently reported another non-moon TTV signal for KOI-314, in this case due to interaction between two planets where both transit their host star. In this case, the outer planet, KOI-314c, was found to have a mass of $M_{P}=1.0_{-0.3}^{+0.4} M_{\oplus}$, making it the lowest mass transiting planet discovered to date. These cases highlight the exciting ancillary science resulting from exomoon surveys.

\section{OUTLOOK}

"When will we discover an exomoon?" is probably the question I get asked the most when discussing this subject, yet a definitive prediction cannot be made for the fundamental reason that the occurrence rate of large moons is presently unknown. Nevertheless, I consider here the future outlook and prospects for success.

The Kepler data undoubtedly presents the only viable channel for detecting transiting exomoons with past or present observatories. The 4+year baseline of continuous, high-precision photometry makes it greatly superior to CoRoT, HST or MOST data collected to date. The lower temporal baseline of the planned TESS (launch 2017-2018) mission makes it less attractive than Kepler for detecting exomoons, but PLATO 2.0 (launch 2024) may offer opportunities with a planned 2 year baseline at Kepler-like precision. JWST will also be too competitive to acquire the large temporal baselines necessary. It therefore appears that if Kepler is unable to succeed in discovering an exomoon, future missions (up to the mid-2020's) will be generally less suitable.

With this point in mind, what are the prospects of Kepler succeeding? The failure of a second reaction wheel ended the nominal Kepler mission. A planned K2 mission will exploit a two-wheel Kepler, but since it uses radiation pressure as a third torque, pointing is limited to near the ecliptic plane, making a re-observation of the original Kepler field impossible. In this sense, the 4.35 years of archival Kepler data represent our best hope for a discovery. Whilst further data would of course benefit the exomoon hunt, 4.35 years is sufficient to identify more than half-a-dozen transits of planets with periods of 8 months or less, which should be sufficient to identify a large moon. 
The HEK project has surveyed just 17 targets to date, yet $\sim 250$ planetary candidates are estimated to have the correct properties to maintain a detectable exomoon. In general, issues with planet false-positives, time-correlated noise and stellar activity limit the feasible sample to a number less than 250. Despite this, at least 100 targets should provide the required data quality to seek exomoons and the HEK project intends to survey all of these over the next 2-3 years.

What kind of sensitivity level should we expect these "good" targets to yield? The light curve fits directly reveal $\left(M_{S} / M_{P}\right)$ and the typical constraints obtained to date (Figure 4) vary considerably from system to system, depending upon the transit profile shape, orbital period, noise properties of the data and presence of perturbing planets in the system. Although only 17 objects have been surveyed, one can use this sample to estimate the approximate sensivity of the HEK survey.

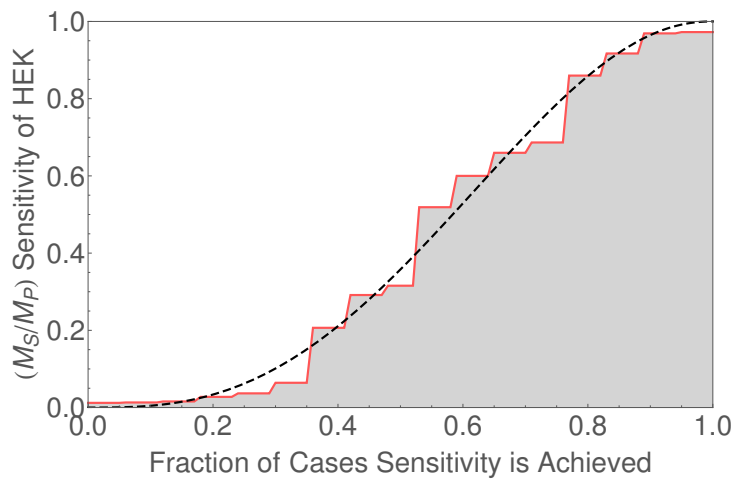

Figure 5: Empirical sensitivity of the HEK project to the satellite planet mass ratio (red line), based on 17 published results. HEK is able to detect Earth-Moon like mass ratios in $\sim 14 \%$ of cases. Black line shows a smoothed functional fit.

Figure 5 shows the mass-ratio sensitivity as a function of the fraction of cases in which the HEK project achieves the said sensitivity. Using the very flexible cumulative density Beta function provides a reasonable smooth fit through the results to date. This function implies HEK is able to achieve the Earth:Moon mass ratio $(=0.012)$ in $\sim 14 \%$ of cases and a Pluto:Charon mass ratio of $(=0.116)$ in $\sim 32 \%$ of cases. If such quasi-binary planet-moon systems are common, then HEK should expect to find them after sampling $\mathscr{O}\left[10^{2}\right]$ systems.

In conclusion, whether HEK discovers several moons or none at all, the results will reveal just how unique the Solar System is. If large moons are common, then temperate satellites with atmospheres and perhaps even life may be a frequent occurrence. If large moons are exceptionally rare, then life in the Universe may be mostly bound to planets and even Earth-Moon quasi-binaries would be a rarity, which may have important implications for the uniqueness of our home world. Either way, though it pushes the instrument to its very limits, Kepler can deliver the answer. It may take more time and analysis than discovering planets, but the occurrence rate of exomoons lies buried within the Kepler data. Its beyond-the-grave determination will shape how we think about the uniqueness of own home and our place in the Universe. 


\section{References}

[1] Campbell B., Walker G. A. H. \& Yang. S. 1988, ApJ, 331, 902

[2] Latham D. W. et al. 1989, Nature, 339, 38

[3] Wolszczan, A. \& Frail, D. 1992, Nature, 355, 145

[4] Mayor, M. \& Queloz, D. 1995, Nature, 378, 355

[5] Marcy, G. W. \& Butler, P. R. 1998, ARA\&A, 36, 57

[6] Batalha, N. et al. 2013, A\&A, 204, 24

[7] Marcy, G. W. 2014, AAS Meeting \#223, \#91.03

[8] Youdin, A. N. \& Kenyon, S. J. 2013, "From Disks to Planets" in T. D. Oswalt, L. M. French, and P. Kalas, editors, Planets, Stars and Stellar Systems. Volume 3: Solar and Stellar Planetary Systems.

[9] Fressin, F. et al. 2013, ApJ, 766, 81

[10] Petigura, E. A., Howard, A. W. \& Marcy, G. W. 2013, PNAS, 110, 19273

[11] Rodler, F. \& López-Morales, M. 2014, ApJ, 781, 54

[12] Gong, Y.-X. et al. 2013, ApJ, 769, L14

[13] Williams, D. M., Kasting, J. F. \& Wade, R. A. 1997, Nature, 385, 234

[14] Heller, R. 2012, A\&A, 545, L8

[15] Heller, R. \& Barnes, R. 2012, Astrobiology, 13, 18

[16] Forgan, D. \& Kipping, D. M. 2013, MNRAS, 432, 2994

[17] Laskar J., Joutel, F. \& Robutel, P. 1993, Nature, 361, 615

[18] Mosqueira, I. \& Estrada, P. R. 2003a, Icarus, 163, 198

[19] Canup, R. M. \& Ward, W. R. 2002, AJ, 124, 3404

[20] Canup, R. M. \& Ward, W. R. 2006, Nature, 441, 834

[21] Canup, R. M. \& Ward, W. R. 2009, in Europa, edited by R. T. Pappalardo, W. B. McKinnon, and K. Khurana, University of Arizona Press, Tucson, pp. 59

[22] Tanaka, H., Takeuchi, T. \& Ward, W. R. 2002, ApJ, 565, 1257

[23] Sasaki, T., Stewart, G. R. \& Ida, S. 2010, ApJ, 714, 1052

[24] Mosqueira, I. \& Estrada, P. R. 2003b, Icarus, 163, 232

[25] Estrada, P. R. et al. 2009, in Europa, edited by R. T. Pappalardo, W. B. McKinnon, and K. Khurana, University of Arizona Press, Tucson, pp. 27

[26] Lin, D. N. C., Bodenheimer, P. \& Richardson, D. C. 1996, Nature, 380, 606

[27] Hartman, W. K. \& Davis, D. R. 1975, Icarus, 24, 504

[28] Agnor, C. B. \& Hamilton, D. P. 2006, Nature, 441, 192

[29] Williams, D. M. 2013, Astrobiology, 13, 315

[30] Porter, S. B. \& Grundy, W. M. 2011, ApJ, 736, L14 
[31] Barnes, J. W. \& OâĂŹBrien, D. P. 2002, ApJ, 575, 1087

[32] Domingos, R. C., Winter, O. C. \& Yokoyama, T. 2006, MNRAS, 373, 1227

[33] Namouni, F. 2010, ApJ, 719, L145

[34] Seager, S. \& Mallén-Ornelas, G. 2003, ApJ, 585, 1038

[35] Seager, S. \& Sasselov, D. D. 2000, ApJ, 537, 916

[36] Russell, H. N. 1948, Harvard Coll. Obs. Monograph, 7, 181

[37] Lewis, K. M., Sackett, P. S. \& Mardling, R. A. 2008, AJ, 685, L153

[38] Kalas, P. et al. 2008, Science, 322, 1345

[39] Bennett, D. P. et al. 2014, ApJ, submitted

[40] Sartoretti, P. \& Schneider, J. 1999, A\&AS, 134, 553

[41] Agol, E. et al. 2005, MNRAS, 359, 567

[42] Holman, M. J. \& Murray, N. W. 2005, Science, 307, 1288

[43] Kipping D. M. 2011, PhD Thesis, University College London

[44] Kipping D. M. 2009a, MNRAS, 392, 181

[45] Kipping D. M. 2009b, MNRAS, 396, 1797

[46] Nesvorný, D. et al. 2013, ApJ, 777, 3

[47] Awiphan, S. \& Kerins, E. 2013, MNRAS, 432, 2549

[48] Kipping D. M., Fossey, S. J. \& Campanella, G. 2009, MNRAS, 400, 398

[49] Ford, E. B. et al. 2012, ApJ, 750, 18

[50] Mandel, K. \& Agol, E. 2002, ApJ, 580, L171

[51] Simon, A. E. et al. 2012, MNRAS, 419, 164

[52] Fewell, M. (2006) Tech. Rep. DSTO-TN-0722 (available online at http://hdl.handle.net/1947/4551).

[53] Rabus, M. et al. 2009, A\&A, 494, 391

[54] Hirano, T. et al. 2012, ApJ, 759, L36

[55] Lissauer, J. J. et al. 2013, ApJ, 770, 131

[56] Carter, J. A. et al. 2011, Science, 331, 562

[57] Kipping D. M. et al. 2012, ApJ, 750, 115

[58] Szabó, Gy. M. et al. 2006, A\&A, 450, 395

[59] Sato, M. \& Asada, H. 2009, PASJ, 61, L29

[60] Sato, M. \& Asada, H. 2010, PASJ, 62, 1203

[61] Tusnski, L. R. M. \& Valio, A. 2011, ApJ, 743, 97

[62] Kipping D. M. 2011, MNRAS, 416, 689

[63] Feroz, F. \& Hobson, M. P. 2008, MNRAS, 384, 449

[64] Feroz, F., Hobson, M. P. \& Bridges, M. 2009, MNRAS, 398, 1601 
[65] Kipping D. M. et al. 2013b, ApJ, 777, 134

[66] Nesvorný, D. et al. 2012, Science, 336, 1133

[67] Kipping D. M. et al. 2013a, ApJ, 770, 101

[68] Kipping D. M. et al. 2014, ApJ, In Press

[69] Weiss, L. M. \& Marcy, G. W. 2014, ApJ, 783, L6 